much it will cost and what sort of effort in terms of manpower and technological research will be needed. The study normally takes three months.

Phase A or study stage. Those proposals not thrown out after the assessment stage are subjected to a more detailed definition. This is usually done under the direction of a technical study team which may contract out to industry some parts of the study. It involves a detailed estimate of the cost and time to completion and a thorough description of the payload, spacecraft, orbit, ground support and data handling and analysis. Typically the study stage lasts nine months and costs $1 \%$ of the final project cost.

Phase $B$ or design stage. A scientific project which enters this stage has usually been approved for development by the SPC. It is put under the management of a project team which will be responsible for it until it has been launched and is operating. The project team places contracts in industry for the design of the spacecraft. Typically, phase B takes one year and costs $10 \%$ of the final cost of the project.

Phase C/D or development and production. Typically, for a scientific satellite, this will take three years and cost 40-70 mau.

The project teams include engineers from ESTEC's department of development and technology (DDT). When they are not part of a project team they do research in one of the DDT's divisions on various aspects of advanced space technology. This research is important for keeping Europe in the forefront of space technology.

Before launch all ESA satellites are tested at ESTEC. It has the only facility in Europe-a high vacuum chamber $14 \mathrm{~m}$ high and $10 \mathrm{~m}$ in diameter-large enough to test a satellite with its booms deployed or the nose cone of Ariane. Once the satellite is in orbit, however, operational responsibility for it shifts to the European Space Operations Centre (ESOC) at Darmstadt, West Germany. As well as control of the orbit, ESOC is responsible for setting up ground stations to receive data.

One of ESA's objectives is to encourage development of European industry. This is reflected in the way it puts out tenders to industry for its scientific satellites. Its industrial policy states that each member state should receive the same percentage of the total contract put out to tender as it contributes to the mandatory science budget. This is irrespective of whether it is participating in the scientific payload. This policy is controlled by the Industrial Policy Committee (IPC) which can suggest how contracts should be placed to ensure that each country gets a fair return.

ESA'S SCIENTIFIC RECORD

\section{The past ...}

ESA and its predecessor ESRO have launched twelve scientific satellites since the first launch in May 1968. Six of them are still operating: $\operatorname{Cos} \mathbf{B}$, a highly successful gamma ray observatory launched in April 1975; Geos 1, which failed to reach geostationary orbit in April 1977 but is producing some useful magnetospheric data; ISEE-2, which was launched in October 1977 and is observing the interaction of the solar wind and the Earth's magnetosphere in conjunction with NASA's ISEE-1; Meteosat, launched in November 1977, contributing to the Global Atmospheric Research Programme and the World Weather Watch; International Ultraviolet Explorer, the ultraviolet astronomical observatory, launched as a NASA-UK-ESA collaboration in January 1978; and Geos 2, a 'copy' of Geos 1, successfully launched into geostationary orbit in June 1978 to observe the magnetosphere.

Of the previous seven satellites, five were designed for magnetospheric and atmospheric experiments, and two for high energy astronomy: ESRO-II for cosmic rays and solar X-rays, launched May 1968) and TD-1 (a UV satellite launched March 1972 and operational for two years but troubled by malfunctioning tape recorders). Of the complete list of thirteen satellites, nine were for Earth-related observations (mostly on Sun-Earth interaction) and four for astronomy. Astronomical satellites were launched in 1968, 1972, 1975 and 1978.

\section{The present ... .}

ESA's report to the 21st COSPAR meeting in June 1978 says that while Sun-Earth relationships "continue to be an important feature of the agency's scientific programme", there is a change afoot: "an increased emphasis is being placed on astronomical studies". This is reflected to some extent in the current list of approvals (four main projects, two of which are astronomical), and to a greater extent among the competing proposals for the next project (three out of five are for astronomy). Approved are:

The first Spacelab payload. Spacelab is scheduled to fly with the tenth flight of the Space Shuttle on 3 December 1983. It will be manned, but will stay aloft for only 7-30 days. According to ESA's 1978 report to COSPAR "the composition of a typical Spacelab payload will be distinctly different from that of a conventional research satellite". Life sciences and materials sciences will benefit from 'look-see' experiments in a new environment. But the observing time is too short for much astronomy.

EXOSAT. This $0.1 \mathrm{keV}$ to $50 \mathrm{keV}$ $\mathrm{X}$-ray astronomy satellite is scheduled for launch on Ariane in February or March 1981 (or by Thor-Delta if Ariane is not ready). It has two operating modes: occultation, using the Earth or Moon to determine the position and structure of X-ray sources, and arbitrary pointing, which will be used to determine this spectral and temporal variation.

Out of Ecliptic/Solar Polar Mission. This is a joint mission with NASA to launch two spacecraft in February 1983 , to fly out of the plane of planetary orbits and around the North and South poles of the Sun. The gravitational field of Jupiter will be used to swing the spacecraft out of the ecliptic. The objective is to extend into three dimensions our knowledge of the solar corona, the solar wind, the solar magnetic field, the cosmic ray flux, and interplanetary/interstellar neutral gas and dust.

The space telescope. This, as ESA's latest report to COSPAR says, "is the most ambitious project in space astronomy currently planned and will dominate astronomical research for the rest of the century". ESA is participating at a level that will allow Europe's astronomers just $15 \%$ of the observing time on the high-resolution, 2.4 metre telescope. The telescope should be launched from the space shuttle at the end of 1983 with five separate focal plane instruments. In a $500-\mathrm{km}$ high orbit, it will be retrievable and refurbishable, and should serve astronomers for two decades.

\section{The future ...}

THE European Space Research and Technology Centre (ESTEC) in the Netherlands is investigating the scientific and technical merit of several spacecraft which will compete for selection as the scientific project to follow the ESA/NASA space telescope which is due for launch in 1983.

To effectively use the science budget ESA's Science Policy Committee (SPC) will have to decide on which experiment to launch by the beginning of 1980 . The next mission should then be ready for launch in 1985 .

The options are already known. According to Dr D. E. Page, head of the science department at ESTEC, any new mission proposals could not be studied in time for next year's decision 

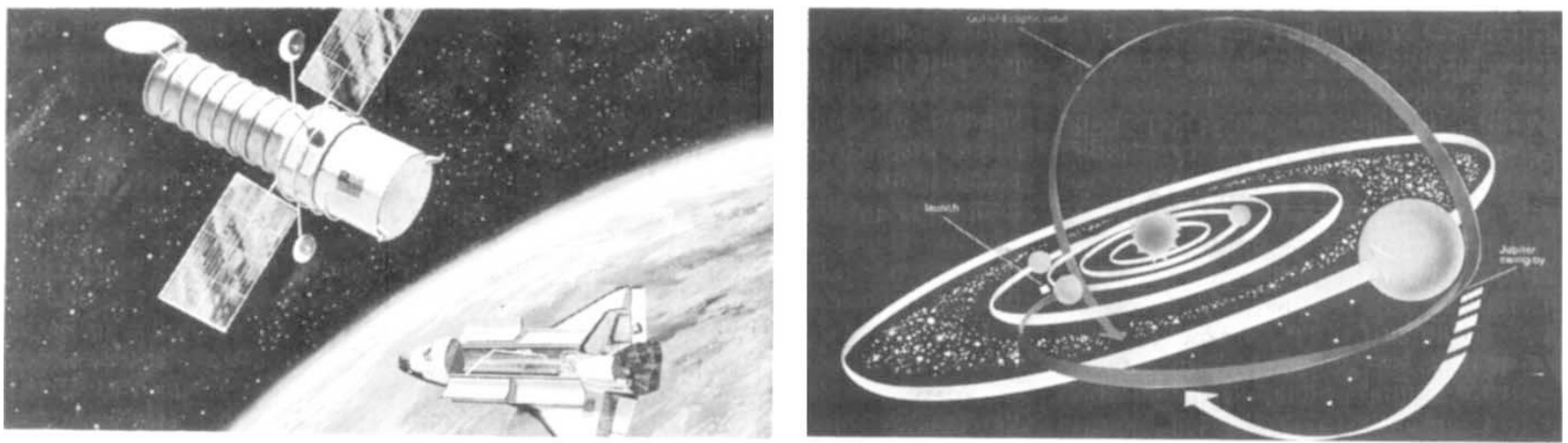

Already approved: space telescope, to be launched from the NASA shuttle, and the Out-of-Ecliptic mission, both scheduled for 1983.

because available staff at ESTEC are already committed. The selection will, therefore, be from the following:

Extreme ultraviolet and $X$-ray survey satellite (EXUV). The proposal for this satellite stemmed from observations made in 1975 by NASA's X-ray satellite, Copernicus. Before that it had been thought that extreme UV radiation was too heavily absorbed by the interstellar medium (ISM) to be observed. Copernicus, however, showed that the local ISM is thinner than had been expected and that in certain directions the emissions can be observed from objects up to 100 parsec away.

ESA's EXUV satellite would survey the sky in the waveband $15 \AA-1,000 \AA$ with a few arc min resolution. It would provide information on very hot stars, which emit most of their radiation in the EUV waveband (250 $\AA$ $1,000 \AA$ ), and stellar coronae. It would also help study the origins of the diffuse XUV $(15 \AA-250 \AA)$ background.

The estimated cost on completion of an EXUV satellite is 75 mau. This includes half an Ariane launch.

Astrometry satellite. The position of stellar objects can be measured with far greater precision from space than from Earth. In principle, positional accuracy could be improved by a factor of 20 and the number of observable stars could rise by a factor of 10 to 100 .

The idea for an astrometry satellite in ESA is about nine years old. However, it has never been one of the candidates for final selection because previous studies have been abandoned at the mission definition stage. One of the problems is that it could be technically very demanding to achieve the claimed increases in accuracy. Because of this, an astrometry satellite could be expensive, of the order of 95 mau.

Grazing-incidence solar telescope (GRIST). This would be an ESAfunded Spacelab facility to observe the Sun in the soft $X$-ray and UV wavebands $(100 \AA-1,700 \AA)$. The advantage of using Spacelab (rather than flying GRIST separately) is that the telescope could be flown as one of several solar telescopes, making simultaneous obser- vations in a variety of wavelengths. NASA is considering building the other telescopes.

The option of another large Spacelab facility, the large infrared telescope (LIRTS), has recently been shelved on cost grounds. Interest in focal plane instrumentation for LIRTS, however, is still being maintained at ESTEC in case the project is ever reconsidered as a free-flyer. The major disadvantage of putting a heavy instrument on Space$l a b$ is that the launch cost for a $7-30$ day flight is as much as the cost of launching it as a free-flyer with an observing time of several years.

Sun-Earth observatory and climatology satellite (SEOCs). This would measure the Earth's radiation budget more accurately than previous NASA satellites. It would help investigate the physical processes controlling the budget by monitoring spatial and spectral changes in the incoming and outgoing radiation. In particular it would monitor the incoming solar UV flux in conjunction with the composition of the stratosphere and observe interactions between different atmospheric levels and the Earth's surface.

SEOCs would be put into circular orbit at a height of $1,150 \mathrm{~km}$ and $57.5^{\circ}$ inclination. It would only be able to improve on existing measurements if it were to interact with geostationary satellites, such as Meteostat, to obtain information on the diurnal characteristics of the atmosphere. It would also have to interact with polar orbiting spacecraft if it were to study the complete radiation budget. Because the usefulness of SEOCs would depend so heavily on it interacting with other spacecraft, ESA is looking at the possibility of building two.

The cost on completion of one satellite plus the cost of half an Ariane launch is estimated to be at least 85 mau. However, ESA has not yet decided whether to fund climatology out of the mandatory science budget or as an optional applications programme. So SEOCs could be removed from the list of candidates for the next scientific mission and be funded separately.
Polar orbiting lunar observatory (POLO). In spite of NASA's Apollo and Lunar missions, there are still fundamental questions about the Moon left unanswered: for example does it have a core, what is its internal temperature, what is its thermal history, and what was the origin of the magnetic field of the early Moon? Some scientists feel these questions would be better answered with the aid of an orbiting spacecraft than a lander, such as Apollo, which is necessarily very limited in the area it can observe.

A study is currently being rushed through at ESTEC to look at the possibility of building POLO very cheaply from Geos 3 hardware and launching it on the fourth Ariane test flight at the end of 1980 . This would mean that POLO could be built cheaply and quickly. It might even mean that it could be funded in addition to one of the other options. The possibility of this happening, however, has grown more remote as cost estimates have risen from a few mau to well above 10 mau.

The SAC will not have to decide which project should follow the one to be approved at the end of next year until the end of 1980. ESA, however, will be sending out a call for mission proposals for that decision in the middle of next month. No one yet knows what that will bring but there is growing pressure from planetary scientists in Europe for ESA to launch spacecraft out of Earth orbit to other parts of the solar system. And POLO is not the only idea they have for the future. They are also considering a cometary mission and a mission to the asteroids. At a workshop organised by ESA earlier this year, European scientists came out in favour of a dual mission to comets Halley and Tempel 2. The proposal they agreed on was to fly-by Halley's comet during its next nearest approach to Earth in 1986 and then, following an Earth swing-by, to rendezvous with Tempel 2 in 1988. Both the proposed cometary and asteroid missions would be extremely expensive. The cost for the cometary mission might be about 500 mau. 